\title{
Editorial
}

\section{Promotion of Hand Hygiene: Magic, Hype, or Scientific Challenge?}

\author{
Didier Pittet, MD, MS
}

The compliance of healthcare workers (HCWs) with hand hygiene practices is universally low. ${ }^{1}$ The challenge of promotion of hand hygiene could be summarized in two simple questions: (1) How can we change the behavior of HCWs? and (2) How can we maintain such change? Predisposing, enabling, and reinforcing factors should be taken into account for the successful promotion of hand hygiene and prevention of nosocomial infections. ${ }^{1-3}$

Among predisposing factors for noncompliance with hand hygiene are physician and nursing assistant status (rather than a nurse), glove wear, skin irritation by hand hygiene agents, and the critical care setting, particularly when heavy workloads exist with an increased number of opportunities for hand hygiene per hour of patient care. ${ }^{1,3,4}$ Time constraint was the most influential parameter in the largest epidemiologic survey conducted. ${ }^{4}$ Self-reported factors for poor adherence with hand hygiene include poor accessibility of sinks or a lack of convenient tools for hand hygiene, lack of knowledge, disagreement with or even skepticism about recommendations, the feeling that hand hygiene might interfere with $\mathrm{HCW}$-patient relations, lack of scientific information reporting a definitive impact of improved hand hygiene on nosocomial rates, the impression that the risk for cross-transmission is low for the patients, the belief that glove use dispenses from hand hygiene, and the idea that patient needs take priority over hand hygiene..$^{1-5}$ Additional key factors for noncompliance are forgetfulness, the lack of a role model in colleagues or superiors, and the absence of an institutional priority for hand hygiene.

On the basis of reported experiences, strategies for the successful promotion of hand hygiene in hospitals include educating $\mathrm{HCWs}$; monitoring $\mathrm{HCW}$ compliance and providing performance feedback; placing reminders in the work place; improving engineering control; promoting and facilitating care of the skin of HCWs' hands; and avoiding overcrowding, understaffing, and excessive workloads. ${ }^{1,3,5,6}$ Other administrative, institutional, and individual measures have been suggested, such as rewarding HCWs who comply, having individual HCWs and institutions actively participate in the promotion of hand hygiene, enhancing individual and institutional self-efficacy, and creating a climate of institutional safety. ${ }^{1-3,5-7}$ Furthermore, ongoing commitment from opinion leaders in the hospital to support the promotion of hand hygiene is fundamental. ${ }^{8}$ Last, but not least, promotion strategies must combine several of these parameters (ie, be multimodal) ${ }^{1-3,5,9}$

Among enabling factors, engineering control must be considered for the successful promotion of hand hygiene. In particular, it involves making hand hygiene easy, convenient, and possible in a timely fashion..$^{1,3,5} \mathrm{New}$ guidelines propose waterless hand antisepsis as the standard for hand hygiene..$^{10}$ Alcohol-based hand rubs are the preferred hand hygiene agents because they reduce bacterial counts on hands more effectively than plain or antimicrobial soaps; can be made more accessible than sinks and other hand washing facilities; require less time for action; and cause less skin irritation and dryness than soap and water. ${ }^{13,6,8,10-13}$ Promoting and facilitating care of the skin of HCWs' hands is part of the recommended strategies.

Although a simple change in the hand hygiene agent being used, such as the introduction of a hand rub, might help, convincing HCWs not only to use it, but to continue using it remains a major challenge. Among reinforcing factors, routine observation of $\mathrm{HCW}$ compliance and performance feedback has been extensively studied and reported..$^{10}$ It is also part of proposed frameworks that list strategies for the successful promotion of hand hygiene..$^{1,3,5,9}$ Although individual, inservice, group, ward, department, and hospital-wide feedback has been used as a 
single strategy, it is most frequently used as part of a multimodal strategy and was considered effective in at least 15 reports. ${ }^{10}$ In this issue of Infection Control and Hospital Epidemiology, Bittner et al. ${ }^{14}$ discuss their extremely careful study to test the hypothesis that sustained feedback will produce a sustained improvement in hand washing. Although sustained feedback on the performance of hand hygiene as a single measure was associated with a transient benefit, it failed to produce a sustained improvement.

Clearly, HCWs' attitudes toward compliance with hand hygiene and the dynamics of behavioral change are complex. The latter involves a combination of education, motivation, and system change., ${ }^{3,10}$ The campaign promoting hand hygiene at the University of Geneva Hospitals constituted the first reported experience of a sustained improvement in compliance with hand hygiene, coinciding with a parallel reduction in nosocomial infections and transmission of methicillin-resistant Staphylococcus aureus. ${ }^{6}$ The promotion of bedside, antiseptic hand rubs largely explained the increase in compliance. The multimodal strategy that contributed to the success of the promotional campaign included repeated monitoring of compliance and performance feedback, communication and education tools, constant reminders in the work environment, active participation and feedback at both individual and organizational levels, and involvement of institutional leaders.

Current behavioral theories and reported experiences also conclude that intervention strategies should be multimodal. ${ }^{1,3,5,9,10}$ However, they are much more difficult to implement and demand more resources than single actions, and it is important to dissect the impact of each component to identify the most effective combination. Importantly, even when easy-to-implement enabling tools are used, single interventions are frequently associated with, at best, transient improvement. ${ }^{15-17}$ The finding of Bittner et al..$^{14}$ of the absence of a significant impact of sustained feedback should certainly not lead to the elimination of this element from frameworks of multimodal intervention strategies, considering the large number of experiences reported and possible associated benefits. ${ }^{10}$

Infection control experts should consider two important additional messages in the study by Bittner et al. ${ }^{14}$ First, and as previously reported, ${ }^{18,19}$ the authors observed a negative correlation between increased patient-to-nurse ratio and decreased compliance with hand hygiene; in other words, the higher the workload, the lower the compliance. Again, a high demand for hand hygiene-a reflection of high workload-is considered a very significant, ${ }^{1,3,4,10-14}$ if not the most significant, ${ }^{4}$ risk factor for noncompliance. In critical care units, this can be corrected by waterless hand antisepsis. ${ }^{20}$

Second, the presence of live observers was associated with an increased frequency of hand washing, even when these observers did not offer feedback on performance. Knowledge of being observed was associated with improved compliance in the study. Observer bias and the
Hawthorne effect are systematically discussed in studies that involved direct observation of hand hygiene practices. My personal opinion is that obtaining a sustained and never-ending Hawthorne effect associated with improved compliance with hand hygiene and decreased infection and cross-transmission rates should be the dream of every hospital epidemiologist. Let's find a cost-effective way to induce it! Could the Orwellian catch phrase "Big brother is watching you" be considered a vital element of the best multimodal approach to ensure a sustained improvement in hand hygiene behavior? The question remains unanswered and certainly merits further investigation.

\section{REFERENCES}

1. Pittet D. Improving compliance with hand hygiene in hospitals. Infect Control Hosp Epidemiol 2000;21:381-386.

2. Kretzer EK, Larson EL. Behavioral interventions to improve infection control practices. Am J Infect Control 1998;26:245-253.

3. Pittet D, Boyce J. Hand hygiene and patient care: pursuing the Semmelweis legacy. Lancet Infectious Diseases 2001;April:9-20.

4. Pittet D, Mourouga $P$, Perneger TV, et al. Compliance with handwashing in a teaching hospital. Ann Intern Med 1999;130:126-130.

5. Pittet D. Improving adherence to hand hygiene practice: a multidisciplinary approach. Emerg Infect Dis 2001;7:234-240.

6. Pittet D, Hugonnet $S$, Harbarth $S$, et al. Effectiveness of a hospital-wide programme to improve compliance with hand hygiene. Lancet 2000;356:1307-1312.

7. Larson EL, Early E, Cloonan P, Sugrue S, Parides M. An organizational climate intervention associated with increased handwashing and decreased nosocomial infections. Behav Med 2000;26:14-22.

8. Harbarth S, Pittet D, Grady L, et al. Interventional study to evaluate the impact of an alcohol-based hand gel in improving hand hygiene compliance. Pediatr Infect Dis $J$ In press.

9. Larson EI, Bryan JL, Adler LM, Blane CB. A multifaceted approach to changing handwashing behavior. Am J Infect Control 1997;25:3-10.

10. Boyce J, Pittet D, for the Centers for Disease Control and Prevention (CDC) Healthcare Infection Control Practices Advisory Committee (HICPAC), the Society for Healthcare Epidemiology of America (SHEA), the Association for Professionals in Infection Control and Epidemiology (APIC), and the Infectious Diseases Society of America (IDSA). HICPAC/SHEA/APIC/IDSA Hand Hygiene Task Force; and the Healthcare Infection Control Practices Advisory Committee. Guideline for hand hygiene in healthcare settings. Federal Register 2001. Available at www.cdc.gov/ncidod/hip/hhguide.htm.

11. Larson E. Skin hygiene and infection prevention: more of the same or different approaches? Clin Infect Dis 1999;29:1287-1294.

12. Boyce JM. Using alcohol for hand antisepsis: dispelling old myths. Infect Control Hosp Epidemiol 2000;21:438-441.

13. Widmer AF. Replace hand washing with use of a waterless alcohol hand rub? Clin Infect Dis 2000;31:136-143.

14. Bittner MJ, Rich EC, Turner PD, Arnold WH Jr. Limited impact of sustained simple feedback based on soap and paper towel consumption on the frequency of hand washing in an adult intensive care unit. Infect Control Hosp Epidemiol 2002;23:120-126.

15. Muto CA, Sistrom MG, Farr BM. Hand hygiene rates unaffected by installation of dispensers of a rapidly acting hand antiseptic. Am J Infect Control 2000;28:273-276.

16. Bischoff WE, Reynolds TM, Sessler CN, Edmond MB, Wenzel RP. Handwashing compliance by health care workers: the impact of introducing an accessible, alcohol-based hand antiseptic. Arch Intern Med 2000;160:1017-1021.

17. Maury E, Alzieu M, Baudel JL, Haram N. Availability of an alcohol solution can improve hand disinfection compliance in an intensive care unit. Am J Respir Crit Care Med 2000;162:324-327.

18. Haley RW, Bregman D. The role of understaffing and overcrowding in recurrent outbreaks of staphylococcal infection in a neonatal specialcare unit. J Infect Dis 1982;145:875-885.

19. Harbarth S, Sudre P, Dharan S, Cadenas M, Pittet D. Outbreak of Enterobacter cloacae related to understaffing, overcrowding, and poor hygiene practices. Infect Control Hosp Epidemiol 1999;20:598-603.

20. Hugonnet S, Perneger TV, Pittet D. Alcohol-based handrub improves compliance with hand hygiene in intensive care units. Arch Intern Med In press. 